\title{
EDITORIAL
}

\section{High altitude and asthma: beyond house dust mites}

\author{
Denis Charpin
}

I $\mathrm{n}$ this issue of the European Respiratory Journal, RIJSSENBEEKNouwENs et al. [1] demonstrated that the benefit of a stay at high altitude was comparable in allergic and nonallergic adult asthmatic patients (allergy was defined on the basis of specific immunoglobulin ( $\mathrm{Ig}) \mathrm{E}$ to a panel of common aeroallergens). This benefit was substantial, exemplified by a discontinuation in oral steroids intake over the course of the stay in about $40 \%$ of both groups of asthmatic patients and an average reduction of $50 \%$ in the other patients.

\section{GENERALLY SPEAKING, THE BENEFIT OF A STAY AT HIGH ALTITUDE IN ALLERGIC ASTHMA IS WELL ESTABLISHED}

The causality of the relationship between decreased exposure to house dust mites (HDM) allergens and asthma control in HDM-allergic patients is indicated through the following evidence: HDM allergen level decreases as altitude increases, apart from in humid climates; asthma control improves [2]; and specific HDM-IgE level [2] and local airway inflammation is reduced during a stay at high altitude [3]. Airway specific as well as nonspecific airway reactivity decreases and goes back to baseline values when the patient returns home [4]. Apart from HDM allergens, exposure to pollen is shorter and lower at high altitude, which, in pollen-allergic patients, accounts for a decrease in seasonal symptoms.

\section{HOW IS A SIMILAR IMPROVEMENT ACCOUNTED FOR IN NON-ALLERGIC ASTHMATIC PATIENTS AT HIGH ALTITUDE?}

The paper suggests that nonspecific air contaminants, whose levels are much lower at high altitude, could play a major role in eliciting asthmatic symptoms, airway inflammation, decreasing pulmonary function and medication requirements. Indeed, major urban air pollutants such as nitrogen oxides and particulate matter are at much lower levels at high altitude and have been shown, over the course of a few hours or days, to be able to increase asthmatic symptoms, decrease pulmonary function, and increase asthma medication requirement. Also, fungi are found in about $30 \%$ of dwellings and often act through nonallergic mechanisms, including primary metabolites, such as glucan, or secondary metabolites, such as mycotoxines and volatile organic compounds [5], and are very unlikely to be present in hospital settings. Besides this microbiological explanation, a physicist's hypothesis lies in the higher air fluidity at high altitude which induces lower airway resistance

Hôpital Nord, Marseille, France.

CORRESPONDENCE: D. Charpin, Clinique des bronches, allergie et sommeil, Hôpital Nord, 13015 Marseille, France. E-mail: denis-andre.charpin@ap-hm.fr and increases inspiratory as well as expiratory air flows. As stated in the paper, this effect is comparable to that of low density gases, such as Heliox, which has been successfully used in the treatment of acute severe asthma.

\section{WHY DO ALLERGIC ASTHMATIC PATIENTS NOT IMPROVE MORE THAN NONALLERGIC ASTHMATIC PATIENTS?}

A first hypothesis may be that there could be a large placebo effect related to moving away during this stay at high altitude from worries and work or family-related conflicts, and to being coddled. These psychological stresses have actually shown to increase maladaptative coping styles in patients with severe asthma [6]. Secondly, a better compliance with medicines, because of implementation of asthma educational programmes and close supervision from the hospital staff during the stay, can be put forward. This hypothesis should be carefully considered knowing that noncompliance affects half to twothirds of asthmatic patients. If this latter hypothesis happened to be true, the benefit from asthma medications could exceed the one linked to the decrease in air contaminants and HDM allergens at high altitude. It would actually be very difficult to get rid of this potential bias. The authors stated that before referring to high altitude, inhalation technique, adherence to treatment, and optimal avoidance of exposure to allergen and irritants had been checked through a questionnaire, but this does not necessarily discard this hypothesis. A possible study design to control this would be to follow-up two groups of asthmatic children during a prolonged stay at high altitude or at sea level, but other environmental differences, such as indoor and outdoor air pollutant level, could still impair the comparison. Thirdly, a review article recently published on the benefit of mite avoidance procedures implemented at home concluded that such a benefit has only been shown in children, but not in asthmatic adults allergic to HDM [5]. Thus, it is not granted that such a benefit demonstrated in both groups could be related only to nonspecific environmental changes. However, in the paper, patients from the allergic asthmatic group showed a larger decrease in total IgE level, blood eosinophils and exhaled nitric oxide fraction, and a somewhat larger corticosteroid discontinuation rate. Besides, the percentage of HDM-allergic patients in the allergy group only equalled $31.5 \%$ and the comparison could have led to a larger decrease in the steroid use in the mite-allergic group would all patients from this group be sensitised to HDM. A fourth hypothesis would be that nonallergic patients, on the basis of serum-specific IgE, could express local IgE sensitisation. Der $p$ specific $\operatorname{IgE}$ were found increased in increased levels in sputum of patients with intrinsic asthma compared to healthy nonatopic subjects, while subjects with intrinsic or atopic 
asthma were indistinguishable in terms of dust mite-specific $\operatorname{IgE}[7]$. However, this $\mathrm{IgE}$ reactivity does not translate into a clinical response to Der $p$ exposure, probably because there is no second signal that promotes IgE-mediated asthmatic responses in intrinsic asthma. Lastly, allergens, especially, mite-allergens could act also through a nonallergic mechanism [8], thus affecting also non-allergic asthmatic patients.

Whatever the correct explanation, the pragmatic result is a major improvement in asthma control at the end of the 12week stay at high altitude, including decrease or even stopping oral corticosteroid use in almost half of this group of severe asthmatic patients. Given the major challenge of controlling the disease, such a result is already rewarding and worthwhile.

Additional studies are indeed needed to replicate these findings, to show if they equally prove true in childhood asthma, to demonstrate if there could be a remanant effect when the patient goes back home and also, together with the general practitioner or nurse, to implement an allergen and irritant-avoiding strategy at home, when the patient is away from home, in order to prepare a safe and healthy environment when he or she is back home.

\section{STATEMENT OF INTEREST}

None declared.

\section{REFERENCES}

1 Rijssenbeek-Nouwens LH, Fieten KB, Bron AO, et al. High-altitude treatment in atopic and nonatopic patients with severe asthma. Eur Respir J 2012; 40: 1371-1377.

2 Vervloet D, Bongrand P, Arnaud A, et al. Objective immunological and clinical data observed during an altitude cure at Briançon in allergic children allergic to house dust and Dermatophagoides. Rev Mal Respir 1979; 7: 19-27.

3 Karagiannidis C, Hense G, Rueckert B, et al. High altitude climate therapy reduces local airway inflammation and modulates lymphocyte activation. Scand J Immunol 2006; 63: 304-310.

4 Peroni DG, Boner AL, Vallone G, et al. Effective allergen avoidance at high altitude reduces allergen-induced bronchial hyperreactivity. Am J Respir Crit Care Med 1994; 149: 1442-1446.

5 Crocker DD, Kinyola S, Dumitru GG, et al. Effectiveness of homebased, multi-trigger, multicomponent interventions with an environmental focus for reducing asthma morbidity: a community guide systematic review. Am J Public Health 2011; 41: S5-S32.

6 Lavoie KL, Bouthillier D, Bason SL, et al. Psychologic distress and maladaptative coping styles in patients with severe vs moderate asthma. Chest 2010; 137: 1324-1331.

7 Mouthuy J, Detry B, Sohy C, et al. Presence in sputum of functional dust-mite specific IgE antibodies in intrinsic asthma. Am J Respir Crit Care Med 2011; 184: 206-214.

8 Roche N, Chinet TC, Belouchi NE, et al. Dermatophagoides pteronyssinus and bioelectric properties of airways epithelium: role of cysteine proteases. Eur Respir J 2000; 16: 309-315. 